\title{
Publisher's Note: General scaling relations for locomotion in granular media [Phys. Rev. E 95, 052901 (2017)]
}

James Slonaker, D. Carrington Motley, Qiong Zhang, Stephen Townsend, Carmine Senatore, Karl Iagnemma, and Ken Kamrin

(Received 31 May 2017; published 7 June 2017)

DOI: 10.1103/PhysRevE.95.069902

This paper was published online on 10 May 2107 with an omission in the Acknowledgments. The Acknowledgments on page 8 should read as "K.K., J.S., and Q.S. gratefully acknowledge support from Army Research Office Grant No. W911NF-15-1-0196 and National Science Foundation Grant No. CBET-1253228. We also acknowledge helpful discussion with staff at the Tank Automotive Research, Development and Engineering Center." The Acknowledgments have been corrected as of 1 June 2017. The Acknowledgments are correct in the printed version of the journal. 